\title{
ASYMPTOTIC DEPTH AND CONNECTEDNESS IN PROJECTIVE SCHEMES
}

\author{
M. BRODMANN
}

(Communicated by William C. Waterhouse)

\begin{abstract}
Let $I \subseteq \mathfrak{m}$ be an ideal of a local noetherian ring $(R, \mathfrak{m})$. Consider the exceptional fiber $\pi^{-1}(V(I))$ of the blowing-up morphism

$$
\pi: \operatorname{Proj}\left(\bigoplus_{n \geq 0} I^{n}\right) \rightarrow \operatorname{Spec}(R)
$$

and the special fiber $\pi^{-1}(\mathfrak{m})$. We show that the complement set

$$
\pi^{-1}(V(I))-\pi^{-1}(\mathrm{~m})
$$

is highly connected if the asymptotic depth of the higher conormal modules $I^{n} / I^{n+1}$ is large.
\end{abstract}

\section{INTRODUCTION}

Let $(R, \mathfrak{m})$ be a local noetherian ring and let $I \subseteq R$ be an ideal of height $>0$. Then it is known that the depths of the $R$-modules $R / I^{n}$ and $I^{n} / I^{n+1}$ take constant values $t(I)$ resp. $\bar{t}(I)$ for all large $n$ [2]:

$$
\begin{aligned}
& \text { (i) } \operatorname{depth}\left(R / I^{n}\right)=t(I) \quad \forall n \gg 0, \\
& \text { (ii) } \operatorname{depth}\left(I^{n} / I^{n+1}\right)=\bar{t}(I) \quad \forall n \gg 0 .
\end{aligned}
$$

$t(I)$ and $\bar{t}(I)$ are called the asymptotic depths of $R / I^{n}$ resp. of $I^{n} / I^{n+1}$. In [3] we have shown

$$
\bar{t}(I) \geq t(I) .
$$

It turns out that these asymptotic depths are related to the topology of the blowing-up of $\operatorname{Spec}(R)$ at $I$, which by definition is given by the canonical morphism

$$
\mathrm{Bl}(I):=\operatorname{Proj}(\mathfrak{R}(I)) \stackrel{\pi_{l}}{\longrightarrow} \operatorname{Spec}(R),
$$

where $\mathfrak{R}(I)$ stands for the Rees algebra $\bigoplus_{n \geq 0} I^{n}$ of $I$. It was noticed by Burch [7] that the dimension of the special fiber $\pi_{I}^{-1}(\mathfrak{m})$ of $(1.3)$ is subject to the inequality $\operatorname{dim}\left(\pi_{I}^{-1}(\mathfrak{m})\right)<\operatorname{dim}(R)-\min _{n} \operatorname{depth}\left(R / I^{n}\right)$.

Received by the editors December 29, 1986.

1980 Mathematics Subject Classification (1985 Revision). Primary 13C15, 14E05. 
In [2] we gave an improvement of this, by showing that $\min _{n} \operatorname{depth}\left(R / I^{n}\right)$ may be replaced by the asymptotic depth $t(I)$ of the rings $R / I^{n}$. In fact, we even may replace $\min _{n} \operatorname{depth}\left(R / I^{n}\right)$ by $\bar{t}(I)$ (cf. [3]):

$$
\operatorname{dim}\left(\pi_{I}^{-1}(\mathfrak{m})\right)<\operatorname{dim}(R)-\bar{t}(I) .
$$

So, if the asymptotic depth $\bar{t}(I)$ is large, the special fiber $\pi_{I}^{-1}(\mathfrak{m})$ must be small.

In this note we want to give a further result of this type. We namely shall prove that for large values of $\bar{t}(I)$ the complement

$$
\pi_{I}^{-1}(V(I))-\pi_{I}^{-1}(\mathfrak{m})
$$

of the special fiber in the exceptional fiber $\pi_{I}^{-1}(V(I))$ is highly connected under certain additional conditions (4.8).

In fact we shall give our result in a more general context. Instead of the blowing-up morphism (1.3) we consider an arbitrary projective morphism $\pi: \operatorname{Proj}(S) \rightarrow \operatorname{Spec}(R)$ (induced by a homogeneous $R$-algebra $S$ ). We then choose a noetherian graded $S$-module $M=\bigoplus_{n \in \mathbb{Z}} M_{n}$ and relate the depths of the $R$-modules $M_{n}$ to the connectivity of the sheaf $\mathscr{F}$ which is induced by $M$ on $\operatorname{Proj}(S)$. Using the connectedness criteria for blowing-up given in [5], we immediately will obtain bounds of connectivity for the set (1.5).

\section{ASYMPTOTIC DEPTH IN PROJECTIVE SCHEMES}

Throughout this section let $(R, \mathfrak{m})$ be a local noetherian ring and let $S=$ $R \oplus S_{1} \oplus S_{2} \oplus \cdots\left(S_{n} \neq 0 \forall n \gg 0\right)$ be a homogeneous noetherian $R$-algebra. So we may write $S=R\left[a_{1}, \ldots, a_{r}\right]$ with $a_{1}, \ldots, a_{r} \in S_{1}$. We consider the canonical morphisms

$$
\gamma: \operatorname{Spec}(S) \rightarrow \operatorname{Spec}(R), \quad \pi: \operatorname{Proj}(S) \rightarrow \operatorname{Spec}(R) .
$$

Moreover, let $M=\bigoplus_{n \in \mathbb{Z}} M_{n}\left(M_{n} \neq 0 \forall n \gg 0\right)$ be a finitely generated, graded essential $S$-module.

Let $\mathscr{F}$ be the coherent sheaf $(\neq 0)$ induced by $M$ on $\operatorname{Proj}(S)$

$$
\mathscr{F}:=\tilde{M} \text {. }
$$

Finally we introduce

$$
\operatorname{depth}_{*}(M):=\min \left\{\operatorname{depth}\left(M_{n}\right) \mid n \in \mathbb{Z}\right\} .
$$

(We make use of the convention depth $(0)=\infty$.) Denoting the grade of $M$ with respect to an ideal $I \subseteq S$ by $g(I, M)$ (by definition this is the maximal length of $M$-regular sequences in $I$ ), we also may write

$$
\operatorname{depth}_{*}(M)=g(\mathfrak{m} S, M) .
$$

This is easily seen in expressing depth and grade by the vanishing of local cohomology and observing the natural isomorphisms $H_{\mathrm{m} S}^{i}(M) \cong \bigoplus_{n \in \mathbb{Z}} H_{\mathrm{m}}^{i}(M)$. 
(2.4) Proposition. (i) $\bigcup_{n \in \mathbb{Z}} \operatorname{Ass}\left(M_{n}\right)=\gamma(\operatorname{Ass}(M))$.

(ii) $\operatorname{depth}_{*}(M)=\min \left\{\operatorname{depth}\left(M_{\mathfrak{p}}\right) \mid \mathfrak{p} \in \gamma^{-1}(\mathfrak{m})\right\}$.

Proof. (i) After an eventual localization it suffices to show that $\mathfrak{m}$ belongs to $\operatorname{Ass}\left(M_{n}\right)$ for some $n$ iff it belongs to $\gamma(\operatorname{Ass}(M))$. The first statement is equivalent to $\operatorname{depth}_{*}(M)=0$, whereas the second one means $g(\mathfrak{m} S, M)=0$. This allows to conclude by $(2.3)^{\prime}$.

(ii) The right-hand side of the stated equality is just $g(\mathfrak{m} S, M)$. So we conclude again by $(2.3)^{\prime}$.

(2.5) Proposition. For all sufficiently large $n \in \mathbb{N}$, the following statements are true:

(i) $\operatorname{Ass}\left(M_{n}\right)=\pi(\operatorname{Ass}(\mathscr{F}))$.

(ii) $\operatorname{depth}\left(M_{n}\right)=\min \left\{\operatorname{depth}\left(\mathscr{F}_{x}\right) \mid x \in \pi^{-1}(\mathfrak{m})\right\}$.

Proof. (i) After an eventual extension of $R$ we may assume that $R / \mathfrak{m}$ is infinite. If we consider the points of $\operatorname{Proj}(S)$ as essential prime ideals in $S, \operatorname{Ass}(\mathscr{F})$ is exactly the set of essential members of $\operatorname{Ass}(M)$. So there is an $n_{0} \in \mathbb{Z}$ with $\operatorname{Ass}(\mathscr{F})=\operatorname{Ass}\left(M_{\geq n_{0}}\right)$, where $M_{\geq n_{0}}$ stands for the submodule $\bigoplus_{n \geq n_{0}} M_{n}$ of $M$. Thus, replacing $M$ by $M_{\geq n_{0}}$, we may write $\operatorname{Ass}(\mathscr{F})=\operatorname{Ass}(M)$, hence $\pi(\operatorname{Ass}(\mathscr{F}))=\gamma(\operatorname{Ass}(M))$. Now, by our choice of $M, \operatorname{Ass}(M)$ has only essential members. So none of the (finitely many) $\mathfrak{p} \in \operatorname{Ass}(M)$ contains $S_{1}$. As $R / \mathfrak{m}$ is infinite this allows to choose an element $f \in S_{1}$ which avoids all members of $\operatorname{Ass}(M)$. We thus obtain injections $M_{n} \stackrel{f}{\rightarrow} M_{n+1}$, which show that $\operatorname{Ass}\left(M_{n}\right) \subseteq$ $\operatorname{Ass}\left(M_{n+1}\right), \forall n \in \mathbb{Z}$. Now we conclude by (2.4)(i).

(ii) We make induction on $d_{M}=\min \left\{\operatorname{depth}\left(\mathscr{F}_{x}\right) \mid x \in \pi^{-1}(\mathfrak{m})\right\}$. If $d_{M}=0$, we conclude by (i). If $d_{M}>0$, clearly $\mathfrak{m} \notin \pi(\operatorname{Ass}(\mathscr{F}))$. We thus find an element $a \in \mathfrak{m}$ which avoids all members of $\pi(\operatorname{Ass}(\mathscr{F}))$. By (i), $a$ becomes $M_{n}$-regular for all $n \gg 0$. So for all sufficiently large $n$ we have $\operatorname{depth}\left(M_{n} / a M_{n}\right)=\operatorname{depth}\left(M_{n}\right)-1$. Moreover by our choice of $a$, we have $\operatorname{depth}\left(\mathscr{F}_{x} / a \mathscr{F}_{x}\right)=\operatorname{depth}\left(\mathscr{F}_{x}\right)-1$, thus $d_{M / a M}=d_{M}-1$. So we conclude applying the hypothesis of induction to $M / a M$.

We introduce the following notations:

$$
\text { (i) } \operatorname{Ass}^{*}(M):=\pi(\operatorname{Ass}(\mathscr{F}))=\operatorname{Ass}\left(M_{n}\right) \quad \forall n \gg 0,
$$

(ii) $\operatorname{depth}^{*}(M):=\min \left\{\operatorname{depth}\left(\mathscr{F}_{x}\right) \mid x \in \pi^{-1}(\mathfrak{m})\right\}=\operatorname{depth}\left(M_{n}\right) \quad \forall n \gg 0$.

$\operatorname{Ass}^{*}(M)$ is called the asymptotic set of prime divisors of $M_{n}$ whereas depth $^{*}(M)$ is called the asymptotic depth of $M_{n}$. We notice

$$
\begin{aligned}
& \text { (i) } \operatorname{Ass}^{*}(M)=\gamma\left(\operatorname{Ass}\left(M_{\geq n}\right)\right) \quad \forall n \gg 0, \\
& \text { (ii) } \operatorname{depth}^{*}(M)=\operatorname{depth}_{*}\left(M_{\geq n}\right) \quad \forall n \gg 0 .
\end{aligned}
$$


As an application of our previous results we want to prove

(2.8) Corollary. $\operatorname{dim}\left(\operatorname{Supp}(\mathscr{F}) \cap \pi^{-1}(\mathfrak{m})\right) \leq \operatorname{dim}(\operatorname{Proj}(S))-\operatorname{depth}^{*}(M)$.

Proof. Let $x$ be a generic point of $\operatorname{Supp}(\mathscr{F}) \cap \pi^{-1}(\mathfrak{m})$. By $(2.5)$ (ii) we have $\operatorname{depth}\left(\mathscr{F}_{x}\right) \geq \operatorname{depth}^{*}(M) ;$ thus $\operatorname{dim}\left(\mathscr{O}_{\operatorname{Proj}(S), x}\right) \geq \operatorname{depth}^{*}(M)$. This induces

$$
\operatorname{dim}(\overline{\{x\}}) \leq \operatorname{dim}(\operatorname{Proj}(S))-\operatorname{dim}\left(\mathscr{O}_{\operatorname{Proj}(S), x}\right) \leq \operatorname{dim}(\operatorname{Proj}(S))-\operatorname{depth}^{*}(M),
$$

hence our claim.

(2.9) Remark. (i) Let $(R, \mathfrak{m})$ be noetherian and local and let $I \subseteq \mathfrak{m}$ be an ideal of positive height. Let $\operatorname{Gr}(I)=\bigoplus_{n \geq 0} I^{n} / I^{n+1}$ be the associated graded ring of $I$. Applying (2.5)(ii) with $S=M=\operatorname{Gr}(I)$, we obtain the asymptotic stability of $\operatorname{depth}\left(I^{n} / I^{n+1}\right)$ for $n \gg 0$ (cf. [2, 3]).

(ii) Let $(R, \mathfrak{m})$ and $I \subseteq R$ as in (i). Consider the canonical diagram

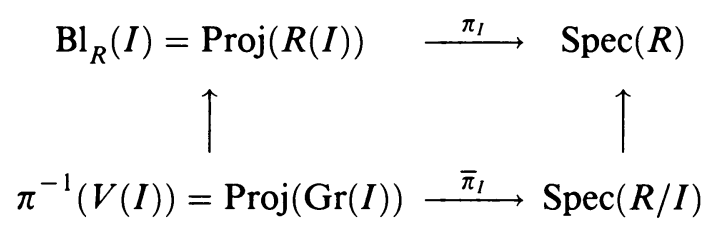

Then, applying (2.8) to $S=M=\operatorname{Gr}(I)$ and observing that $\operatorname{dim}(\operatorname{Proj}(\operatorname{Gr}(I))=$ $\operatorname{dim}(R)-1$ we get $\operatorname{dim}\left(\pi_{I}^{-1}(\mathfrak{m})\right)=\operatorname{dim}\left(\bar{\pi}_{I}^{-1}(\mathfrak{m} / I)\right) \leq \operatorname{dim}(R)-1-\bar{t}(I)$. This proves again (1.4). In the same way we get the asymptotic stability of the sets $\operatorname{Ass}\left(I^{n} / I^{n+1}\right)$ which is shown in [1].

\section{CONNECTEDNESS-SUBdimENSION}

We define the subdimension $\underline{\operatorname{dim}}(Z)$ of a closed set $Z$ in a noetherian scheme $X$ as the minimal codimension (in $Z$ ) of all closed points $x \in Z$. Thus we may write

$$
\underline{\operatorname{dim}}(Z)=\min \left\{\operatorname{dim}_{x}(Z) \mid x \in Z \text { closed }\right\} \quad(\leq \operatorname{dim}(Z)) .
$$

To be complete we define $\operatorname{dim}(\varnothing)=\underline{\operatorname{dim}}(\varnothing)=-1$.

If $Z \subseteq X$ is a closed subset, we define the connectedness-subdimension of $Z$ as follows:

$$
\underline{c}(Z):=\min \{\underline{\operatorname{dim}}(W) \mid W \subseteq Z \text { closed, } Z-W \text { disconnected }\} .
$$

Thereby the empty set $\varnothing$ is considered as disconnected. So we have $\underline{c}(Z) \geq$ -1 with $\underline{c}(Z) \geq 0$ iff $Z$ is connected. Comparing with the connectednessdimension $c(Z)$ introduced as in [6], we obviously have $\underline{c}(Z) \leq c(Z)$. If $Z \neq \varnothing$ we have

$$
\underline{c}(Z)=\min \left\{\underline{\operatorname{dim}}\left(Z_{1} \cap Z_{2}\right)\right\},
$$

where $Z_{1}$ and $Z_{2}$ are unions of irreducible components of $Z$ such that $Z=$ $Z_{1} \cup Z_{2} ; Z_{1}, Z_{2} \neq \varnothing$. Now, let $\mathscr{F} \neq 0$ be a coherent sheaf over $X$. In view 
of (3.2) it is natural to define the connectedness-subdimension of $\mathscr{F}$ by the formula

$$
\underline{c}(\mathscr{F}):=\min \left\{\underline{\operatorname{dim}}\left(\bar{T}_{1} \cap \bar{T}_{2}\right) \mid T_{1} \cup T_{2}=\operatorname{Ass}(\mathscr{F}), T_{1}, T_{2} \neq \varnothing\right\} .
$$

To be complete we define $\underline{c}(0)=-1$. We say that a coherent sheaf $\mathscr{F}$ is connected if $\underline{c}(\mathscr{F}) \geq 0$. Obviously we have

$$
\begin{aligned}
& \text { (i) } \underline{c}(\mathscr{F})=\min \{\underline{c}(\operatorname{Supp}(\mathscr{F})), \operatorname{dim} \overline{\{x\}} \mid x \in \operatorname{Ass}(\mathscr{F})\} \text {, } \\
& \text { (ii) } \mathscr{F} \text { connected } \Longleftrightarrow \operatorname{Supp}(\mathscr{F}) \text { connected. }
\end{aligned}
$$

To relate depths and connectivity for sheaves we prove

(3.5) Lemma. Let $A$ be a noetherian ring, let $I \subseteq A$ be an ideal, and let $M \neq$ 0 be an indecomposable finitely generated A-module with $g(I, M)>1$. Let $T_{1}, T_{2} \subseteq \operatorname{Ass}(M)$ such that $T_{1}, T_{2} \neq \varnothing, T_{1} \cup T_{2}=\operatorname{Ass}(M)$. Then $\bar{T}_{1} \cap \bar{T}_{2} \nsubseteq$ $V(I)$.

Proof. Suppose $\bar{T}_{1} \cap \bar{T}_{2} \subseteq V(I)$. Put $J_{i}=\bigcap_{\mathfrak{p} \in T_{i}} \mathfrak{p} \quad(i=1,2)$. Then $I \subseteq$ $\sqrt{J_{1}+J_{2}}$. In particular we have $g\left(J_{1}+J_{2}, M\right) \geq g(I, M)>1, g\left(J_{i}, M\right)=$ $0(i=1,2)$ and $J_{1} \cap J_{2} \subseteq \sqrt{\operatorname{ann}(M)}$. Considering the following piece of the Mayer-Vietoris sequence for local cohomology:

$$
H_{J_{1}+J_{2}}^{0}(M) \rightarrow H_{J_{1}}^{0}(M) \oplus H_{J_{2}}^{0}(M) \rightarrow H_{J_{1} \cap J_{2}}^{0}(M) \rightarrow H_{J_{1}+J_{2}}^{1}(M),
$$

we get $H_{J_{1}}^{0}(M) \oplus H_{J_{2}}^{0}(M) \cong H_{J_{1} \cap J_{2}}^{0}(M)=M, H_{J_{i}}^{0}(M) \neq 0$. This contradicts the assumption that $M$ is indecomposable.

We also shall need the following graded version of (3.5), which is shown in the same way using "graded" local cohomology.

(3.5)' Lemma. Let $A$ be a noetherian graded ring, let $I \subseteq A$ be a graded ideal, and let $M \neq 0$ be a finitely generated graded A-module, which is indecomposable in the category of graded A-modules. Let $g(I, M)>1$ and let $T_{1}, T_{2} \subseteq \operatorname{Ass}(M)$ such that $T_{1}, T_{2} \neq \varnothing, T_{1} \cup T_{2}=\operatorname{Ass}(M)$. Then $\bar{T}_{1} \cap \bar{T}_{2} \nsubseteq V(I)$.

As an application of (3.5) we get

(3.6) Lemma. Let $X$ be a noetherian scheme and let $\mathscr{F}$ be a connected coherent sheaf over $X$ such that the stalk $\mathscr{F}_{y}$ is an indecomposable $\mathscr{O}_{X, y}$-module for each closed point $y$ of $\operatorname{Supp}(\mathscr{F})$. Let $Z \subseteq X$ be a closed set, which contains all closed points of $\operatorname{Supp}(\mathscr{F})$. Finally assume that $d_{Z}:=\min \left\{\operatorname{depth}\left(\mathscr{F}_{x}\right) \mid x \in Z\right\}>1$.

Then the restriction $\mathscr{F} \mid X-Z$ of $\mathscr{F}$ to the open subset $X-Z$ is connected and satisfies the inequality $\underline{c}(\mathscr{F} \mid X-Z) \geq d_{Z}-2$.

Proof. As $d_{Z}>0$ we have $\operatorname{Ass}(\mathscr{F} \mid X-Z)=\operatorname{Ass}(\mathscr{F})$. Now let $T_{1}, T_{2} \subseteq \operatorname{Ass}(\mathscr{F})$ be nonempty and such that $T_{1} \cup T_{2}=\operatorname{Ass}(\mathscr{F})$. Denote by $\bar{T}_{1}$ and $\bar{T}_{2}$ their closures in $X$. As $\mathscr{F}$ is connected we have $\bar{T}_{1} \cap \bar{T}_{2} \neq \varnothing$. So we find a closed point $y \in \bar{T}_{1} \cap \bar{T}_{2}$. By our assumption we have $y \in Z$, and $\mathscr{F}_{y}$ is indecomposable. Let $I \subseteq \mathscr{O}_{X, y}$ be the vanishing ideal of $\bar{T}_{1} \cap \bar{T}_{2}$ at $y$. By (3.5) we have $g\left(I, \mathscr{F}_{y}\right) \leq 1$. Let $J \subseteq \mathscr{O}_{x, y}$ be the vanishing ideal of $Z$ at $y$ and let 
$\mathfrak{p}$ be a minimal prime divisor of $I+J$. Then clearly we have $g\left(\mathfrak{p}, \mathscr{F}_{y}\right) \geq d_{Z}$. This induces $\operatorname{ht}(\mathfrak{p} / I) \geq d_{Z}-1$; thus $\operatorname{dim}_{x}\left(\bar{T}_{1} \cap \bar{T}_{2}\right) \geq d_{Z}-2$ for all closed points $x$ of $\bar{T}_{1} \cap \bar{T}_{2}-Z$, which satisfy $y \in \overline{\{x\}}$. Making $y$ run through all closed points of $\bar{T}_{1} \cap \bar{T}_{2}$, we obtain $\underline{\operatorname{dim}}\left(\bar{T}_{1} \cap \bar{T}_{2}-Z\right) \geq d_{Z}-2$. This proves our claim.

We now return to the morphism $\pi: \operatorname{Proj}(S) \rightarrow \operatorname{Spec}(R)$ defined in (2.1) and look at the connectivity of the sheaf $\mathscr{F}$ induced by a noetherian, graded, essential $S$-module $M=\bigoplus_{n \in \mathbb{Z}} M_{n}$ (cf. (2.2)). Defining $\operatorname{depth}^{*}(M)$ according to $(2.3)$ we have

(3.7) Proposition. Assume that $\mathscr{F}$ is connected and that the stalks of $\mathscr{F}$ are indecomposable in all closed points of $\operatorname{Supp}(\mathscr{F})$. Let $\operatorname{depth}^{*}(M)>1$. Then the restriction $\mathscr{F} \mid\left(\operatorname{Proj}(S)-\pi^{-1}(\mathfrak{m})\right)$ is connected and satisfies the inequality

$$
\underline{c}\left(\mathscr{F} \mid\left(\operatorname{Proj}(S)-\pi^{-1}(\mathfrak{m})\right)\right) \geq \operatorname{depth}^{*}(M)-2 .
$$

Proof. Apply (3.6) with $Z=\pi^{-1}(\mathfrak{m})$ and use (2.5)(ii).

We notice the following criterion for the connectedness of $\mathscr{F}$, in which $S_{\geq 1}$ denotes the irrelevant ideal $S_{1} \oplus S_{2} \oplus \cdots$ of $S$.

(3.8) Proposition. Let $M$ be indecomposable as a graded module and assume that $g\left(S_{\geq 1}, M\right)>1$. Then $\mathscr{F}$ is connected.

Proof. Let $T_{1}, T_{2} \subseteq \operatorname{Ass}(\mathscr{F})$ nonempty and such that $T_{1} \cup T_{2}=\operatorname{Ass}(\mathscr{F})$. We must show $\bar{T}_{1} \cap \bar{T}_{2} \neq \varnothing(\operatorname{in} \operatorname{Proj}(S))$. Considering $T_{1}$ and $T_{2}$ as sets of prime ideals this comes up to prove that there are homogeneous prime ideals $\mathfrak{q} \in \operatorname{Spec}(S)-V\left(S_{\geq 1}\right), \mathfrak{p}_{1} \in T_{1}, \mathfrak{p}_{2} \in T_{2}$ with $\mathfrak{p}_{1}, \mathfrak{p}_{2} \subseteq \mathfrak{q}$. This is clear by (3.5) '.

(3.9) Remark. The condition $g\left(S_{\geq 1}, M\right)>1$ exactly means that there is a canonical isomorphism (cf. [10])

$$
M \stackrel{\cong}{\rightrightarrows} \bigoplus_{n} H^{0}(\operatorname{Proj}(S), \mathscr{F}(n)) .
$$

This observation also immediately gives a proof of (3.8). In the case $M=S$ the connectivity of $\mathscr{F}=\mathscr{O}_{\operatorname{Proj}(S)}$ exactly corresponds to the connectivity of $\operatorname{Proj}(S)$ (see (3.4)(ii)) and thus is a classical subject of algebraic geometry. One of the mostly used criteria for the connectedness of $\operatorname{Proj}(S)$ is the fact that $\operatorname{Proj}(S)$ is connected iff its ring of global sections $H^{0}\left(\operatorname{Proj}(S), \mathscr{O}_{\operatorname{Proj}(S)}\right)=\Gamma$ is a local ring. This is due to the observation that $\operatorname{Spec}(\Gamma) \stackrel{\nu}{\longrightarrow} \operatorname{Spec}(R)$ is a Grothendieck-Stein factor of the morphism $\pi: \operatorname{Proj}(S) \rightarrow \operatorname{Spec}(R)$ [8].

\section{BLOWING UP}

In this section let $(R, \mathfrak{m})$ be a local noetherian ring and let $I \subseteq \mathfrak{m}$ be an ideal of positive height. We want to study the blowing up morphism (cf. (1.3))

$$
\mathrm{Bl}_{R}(I)=\operatorname{Proj}(\Re(I)) \stackrel{\pi_{I}}{\longrightarrow} \operatorname{Spec}(R) .
$$


Thereby we are mainly interested in connectivity of the exceptional fiber

$$
F_{R}(I):=\pi_{I}^{-1}(V(I))=\operatorname{Proj}(\operatorname{Gr}(I))
$$

of $\pi_{I}$ and of the complement

$$
C_{R}(I):=F_{R}(I)-\pi_{I}^{-1}(\mathfrak{m})
$$

of the special fiber $\pi_{I}^{-1}(\mathfrak{m})$ in exceptional fiber. First we give the following connectedness-criterion for the exceptional fiber $F_{R}(I)$, in which $\hat{R}$ denotes the m-adic completion of $R$.

(4.3) Proposition. If $\operatorname{Spec}(\hat{R})-V(I \hat{R})$ is connected, the exceptional fiber $F_{R}(I)$ is connected.

Proof. Assume that $\operatorname{Spec}(\hat{R})-V(I \hat{R})$ is connected. Let $Z_{1}, Z_{2}$ be unions of irreducible components of $\mathrm{Bl}_{R}(I \hat{R})$ such that $Z_{1} \cup Z_{2}=\mathrm{Bl}_{\hat{R}}(I \hat{R})$. As $\operatorname{ht}(I \hat{R})>0$ the canonical images $\pi_{I \hat{R}}\left(Z_{1}\right), \pi_{I \hat{R}}\left(Z_{2}\right) \subseteq \operatorname{Spec}(\hat{R})$ are again unions of irreducible components and cover the whole set $\operatorname{Spec}(\hat{R})$. By our connectedness assumption $\pi_{I \hat{R}}\left(Z_{2}\right) \cap \pi_{I \hat{R}}\left(Z_{1}\right)$ contains a point $y \in \operatorname{Spec}(\hat{R})-V(I \hat{R})$. In particular, $y$ has one single preimage point $x \in \mathrm{Bl}_{\hat{R}}(I \hat{R})$. So $x$ must belong to $Z_{1} \cap Z_{2}$. This shows that $\mathrm{Bl}_{\hat{R}}(I \hat{R})$ is connected. Therefore $F_{R}(I)$ must be connected (cf. [6, (3.4) or 5, (2.5)]).

(4.4) Corollary. If $g(I, R)>1$, the exceptional fiber $F_{R}(I)$ is connected.

Proof. Passing to completion we get $g(I \hat{R}, \hat{R})>1$. Applying (3.5) with $A=$ $M=\hat{R}$ we see that $\operatorname{Spec}(\hat{R})-V(I \hat{R})$ is connected and thus may conclude by (4.3).

Now we formulate a connectedness-criterion for the complement $C_{R}(I)$. To do so, we introduce the set $T(I)$ of all images of generic points of the exceptional fiber:

$$
T(I):=\left\{\pi_{I}(x) \mid x=\text { generic point of } F_{R}(I)\right\} .
$$

Using this notation we have

(4.6) Proposition. Assume that $g(I, R)>1$ or that $R$ is an excellent normal domain. Then $C_{R}(I)$ is connected if and only if $T(I)$ satisfies the condition

(*) $\bar{U}_{1} \cap \bar{U}_{2}-\{\mathfrak{m}\} \neq \varnothing \quad \forall U_{1}, U_{2} \subseteq T(I)$ with $U_{1}, U_{2} \neq \varnothing$ and $U_{1} \cup U_{2}=T(I)$.

Proof. Clearly the connectedness of $C_{R}(I)$ induces the condition (*). To prove the converse, let $T_{1} \cup T_{2}$ be a decomposition of the set of all generic points of $F_{R}(I)$ such that $\bar{T}_{i}-\pi_{I}^{-1}(\mathfrak{m}) \neq \varnothing(i=1,2)$. It suffices to show that $\bar{T}_{1} \cap \bar{T}_{2}-\pi_{l}^{-1}(\mathfrak{m})$ is not empty. As $T(I)$ satisfies the condition (*), we find a $\mathfrak{p} \in \pi_{I}\left(\bar{T}_{1}\right) \cap \pi_{I}\left(\bar{T}_{2}\right)-\{\mathfrak{m}\}$. We claim that the exceptional fiber $F_{R_{\mathfrak{p}}}\left(I_{\mathfrak{p}}\right)$ of the localized blowing-up $\mathrm{Bl}_{R_{\mathrm{p}}}\left(I_{\mathfrak{p}}\right) \rightarrow \operatorname{Spec}\left(R_{\mathfrak{p}}\right)$ is connected. This follows from (4.4), respectively from (4.3), as either $g\left(I_{\mathfrak{p}}, R_{\mathfrak{p}}\right)>1$ or as $\left(R_{\mathfrak{p}}\right)^{\wedge}$ is a domain (cf. [9]). 
If we consider $F_{R_{\mathrm{p}}}\left(I_{\mathrm{p}}\right)$ as a subset of $F_{R}(I)$, the observed connectedness furnishes a point $x \in F_{R_{\mathfrak{p}}}\left(I_{\mathfrak{p}}\right) \cap \bar{T}_{1} \cap \bar{T}_{2}$. As $F_{R_{\mathfrak{p}}}\left(I_{\mathfrak{p}}\right) \cap \pi_{I}^{-1}(\mathfrak{m}) \neq \varnothing$, we get our claim.

Now, we want to give an estimate on the small connectedness-subdimension $\underline{\dot{c}}\left(C_{R}(I)\right)$ of $C_{R}(I)$, which we define by

$$
\underline{\underline{c}}\left(C_{R}(I)\right):=\underline{c}\left(\mathscr{O}_{\operatorname{Proj}(\operatorname{Gr}(I))} \mid C_{R}(I)\right) \leq \underline{c}\left(C_{R}(I)\right) .
$$

Defining $\bar{t}(I)$ according to (1.1)(ii) we get

(4.8) Proposition. Let $\bar{t}(I)>1$ and assume that either $\operatorname{Spec}(\hat{R})-V(I \hat{R})$ is connected or that $g(I, R)>1$. Then $C_{R}(I)$ is connected and satisfies

$$
\underline{\dot{c}}\left(C_{R}(I)\right) \geq \bar{t}(I)-2 \text {. }
$$

Proof. By (4.3) or by (4.4), $F_{R}(I)$ is connected. Now we apply (3.7) with $S=M=\mathrm{Gr}(I)$.

(4.9) Example. Let $(R, \mathfrak{m})$ be a local Cohen-Macaulay ring and let $\mathfrak{p} \in \operatorname{Spec}(R)$ be an almost complete intersection of height $h>1$, which is a generic complete intersection. Thus, denoting by $\mu_{\mathfrak{q}}(\mathfrak{p})$ the minimal number of generators of the localized ideal $\mathfrak{p}_{\mathfrak{q}} \subseteq R_{\mathfrak{q}}(\mathfrak{q} \in \operatorname{Spec}(R))$, we have $\mu_{\mathfrak{m}}(\mathfrak{p})=h+1, \mu_{\mathfrak{p}}(\mathfrak{p})=h$. We put

$$
U(\mathfrak{p}):=\left\{\mathfrak{q} \in V(\mathfrak{p}) \mid \mathbf{h t}(\mathfrak{q} / \mathfrak{p})=1, \mu_{\mathfrak{q}}(\mathfrak{p})=h+1\right\} .
$$

By [4] we know

(i) $\bar{t}(\mathfrak{p})=\min \{\operatorname{dim}(R / \mathfrak{p})-1, \operatorname{depth}(R / \mathfrak{p})\}$,

(ii) if $\mathfrak{q} \in V(\mathfrak{p}), \tilde{\mathfrak{q}}:=\mathfrak{q} \operatorname{Gr}(I)_{\mathfrak{q}} \cap \operatorname{Gr}(I)$ is the unique minimal prime divisor of $\mathfrak{q} \operatorname{Gr}(I)$ which retracts to $\mathfrak{q}$.

(iii) $\mathfrak{q} \rightarrow \tilde{\mathfrak{q}}$ defines a 1-1 correspondence

$$
U(\mathfrak{p}) \cup\{\mathfrak{p}\} \stackrel{\sim}{\sim}\left\{x \in F_{R}(\mathfrak{p}) \mid x \text { generic }\right\}=\operatorname{Ass}\left(\mathscr{O}_{\operatorname{Proj}(\operatorname{Gr}(\mathfrak{p}))}\right) .
$$

By (4.6) and (4.8) we get from (i) and (iii)

(iv) (a) $C_{R}$ (p) is connected.

(b) $\underline{\dot{c}}\left(C_{R}(\mathfrak{p})\right)=\underline{c}\left(C_{R}(\mathfrak{p})\right) \geq \min \{\operatorname{dim}(R / \mathfrak{p})-3, \operatorname{depth}(R / \mathfrak{p})-2\}$.

\section{REFERENCES}

1. M. Brodmann, Asymptotic stability of Ass $\left(M / I^{n} M\right)$, Proc. Amer. Math. Soc. 74 (1979), 1618.

2. __ On the asymptotic nature of the analytic spread, Math. Proc. Cambridge Philos. Soc. 86 (1979), 35-39.

3. _ Some remarks on blow-up and conormal cones, Proc. Conf. Commutative Algebra (Trento, 1981), Lecture Notes in Pure and Appl. Math., 84, Dekker, New York, 1983.

4. __ Rees rings and form rings of almost complete intersections, Nagoya Math. J. 88 (1982), $1-16$.

5. __ A few remarks on blowing-up and connectedness, J. Reine Angew. Math 370 (1986), 52-60.

6. M. Brodmann and J. Rung, Local cohomology and the connectedness dimension in algebraic varieties, Comment. Math. Helv. 61 (1986), 481-490.

7. L. Burch, Codimension and analytic spread, Proc. Cambridge Philos. Soc. 72 (1972), 369-373. 
8. A. Grothendieck, EGA. III, Inst. Hautes Études Sci. Publ. Math. 11 (1961).

9. __ EGA. IV, Inst. Hautes Études Sci. 24 (1969).

10. J. P. Serre, Faisceaux algébriques cohérents, Ann. of Math. 61 (1955), 197-278.

Mathematisches InStitut der Universität, RÄmistrasse 74, CH-8001 Zuerich, SwitzerLAND 